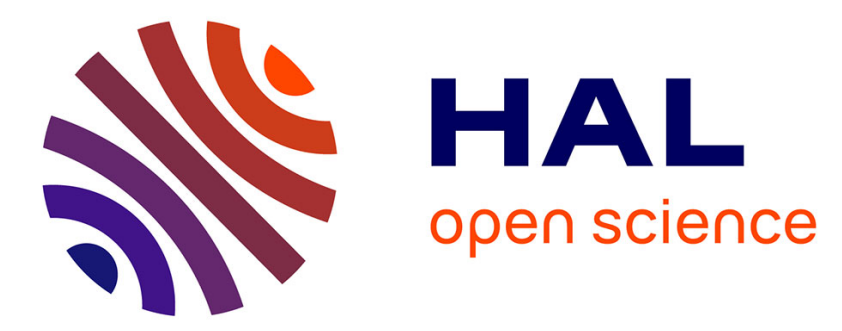

\title{
Modeling the early ionization of dielectrics by ultrashort laser pulses
}

\author{
Antoine Bourgeade, Candice Mézel, Olivier Saut
}

\section{To cite this version:}

Antoine Bourgeade, Candice Mézel, Olivier Saut. Modeling the early ionization of dielectrics by ultrashort laser pulses. Journal of Scientific Computing, 2010, 44, pp.170-190. 10.1007/s10915-0109375-0 . hal-00489482

\section{HAL Id: hal-00489482 https://hal.science/hal-00489482}

Submitted on 4 Jun 2010

HAL is a multi-disciplinary open access archive for the deposit and dissemination of scientific research documents, whether they are published or not. The documents may come from teaching and research institutions in France or abroad, or from public or private research centers.
L'archive ouverte pluridisciplinaire HAL, est destinée au dépôt et à la diffusion de documents scientifiques de niveau recherche, publiés ou non, émanant des établissements d'enseignement et de recherche français ou étrangers, des laboratoires publics ou privés. 


\title{
Modeling the early ionization of dielectrics by ultrashort laser pulses
}

\author{
Antoine Bourgeade* $\quad$ Candice Mézel ${ }^{\dagger} \quad$ Olivier Saut $^{\ddagger}$
}

\begin{abstract}
In this paper, we present a model for propagation of intense and ultrashort laser pulses ionizing dielectrics. We consider early ionization so that this process is sufficiently weak to avoid requiring a complete description of the ionization process (e.g. the use of kinetic equations which are very expensive from a computational point of view). As the intensity of the field is small, one photon ionization is neglected. Ionization may only occur through multi-photonic and collisional ionization. The conduction band is discretized and multiple-rate equations are written for electron densities. The wavefield evolves through Maxwell equations.

Keywords: Nonlinear optics ; Modeling multiphoton ionization ; Ultrashort laser pulses ; Plasma Physics.
\end{abstract}

\section{Introduction}

In this paper, we present a mathematical model adequate to describe the interaction of dielectrics with ultrashort laser pulses. This interaction involves several physical mechanisms. Provided that the laser intensity is high enough (about tens of $\mathrm{TW} / \mathrm{cm}^{2}$ ), electrons are generated by multiphoton ionization (MPI). They reach the conduction band where they get enough energy (more than the band gap value) to perform electronic collisional ionization. One can see $[3,9]$ for the coupling of MPI with a propagating laser field (using the envelope approximation).

There are two other ways to perform ionization: the tunnel ionization, often competing with MPI in which the electrons cross the coulombian

\footnotetext{
${ }^{*}$ CEA/CESTA, B.P. 2, 33114 Le Barp, France, (Antoine.BOURGEADE@cea.fr)

†CELIA, 351 cours de la libération, 33405 Talence Cedex, France, (mezel@celia.u-bordeaux1.fr)

${ }^{\ddagger}$ Institut de Mathématiques, CNRS UMR 5251 and INRIA Bordeaux Sud-Ouest EPI MC2, Université Bordeaux 1, 351 cours de la libération, 33405 Talence Cedex, France, (Olivier.Saut@math.u-bordeaux1.fr)
} 
barrier instead of jumping over, and the above threshold ionization (ATI) in which the electrons get more than the number of photons required for MPI. These two processes are not described in this paper but could easily be included in the proposed method.

In this paper, we couple the propagation of the pulse described by Maxwell equations and a multigroup approach inspired from [19]. Thus we believe that the accounting of the ionization process will be more precise than for instance in [16] where a temperature model of ionization is coupled with Maxwell equations. As we do not make the envelope hypothesis, the interaction will be more precisely described than in [8] where a multigroup model is coupled with an envelope equation for the propagation.

Femtosecond laser pulses can now be used for precise machining at nanometer scales [22]. Indeed these pulses produce limited collateral damages in terms of stress waves, thermal conduction or melting. Ultrashort beams also permit to observe very short phenomena (chemical reaction in gases...). Their enormous power allows them to be used for material ablation or nanomachining $[10,17]$ but does not prevent their application to biological materials [12].

There are many approaches to describe this interaction. One can find models using kinetic approaches based on Fokker-Plank equations [1, 21] or even physical models with an absorption term. However, these approaches are very computationally expensive. We are looking at modeling early ionization where only a small part of the material is ionized. This model should be numerically efficient and adequate to describe multi-photonic ionization. We are not really interested in rendering strong ionization.

On this topic of weak ionization, one can already find several models. For instance in [16], free electrons are considered as a unique variable. The wave-field is driven by Maxwell equations. Two ionization models are introduced : multi-photonic and collisional ionizations. The collisional model is based on a temperature to compute collisional ionization rate. This assumes that the electrons have reached a thermal equilibrium, which may not be the case.

In [18], a multi-group approach is used through multiple rate equations. However, the evolution of the electric field is not accounted for. The ionization equations are solved in the time domain at a given spatial location for a given profile of the laser pulse. Furthermore, some probabilities describing one photon absorption by an electron are difficult to obtain or estimate experimentally.

In this paper, we have combined both approaches. For this matter, several assumptions are made. The displacement of free electrons is neglected as we are interested in weak ionization and short pulses. Finally, the electrons ionizing by the collision process turn back to the lowest energy level of the conduction band. The ionization model is coupled with a Maxwell 
equations solver. This allows us to obtain a model adapted to the early ionization of dielectrics by ultrashort laser pulses.

The outline of this paper is as follows. Section 1 describes the mathematical model. Section 2 describes the numerical methods and algorithms that were used to discretize this model. Finally, Section 3 presents some numerical experiments highlighting the accuracy of our model.

\section{Theoretical model}

In our model, we consider several phenomena affecting electrons and neutrals. It includes multiphoton and collisional ionization, ohmic heating of free electrons by the laser pulses, radiation (excitons) and collisions between electrons and electrons/neutrals.

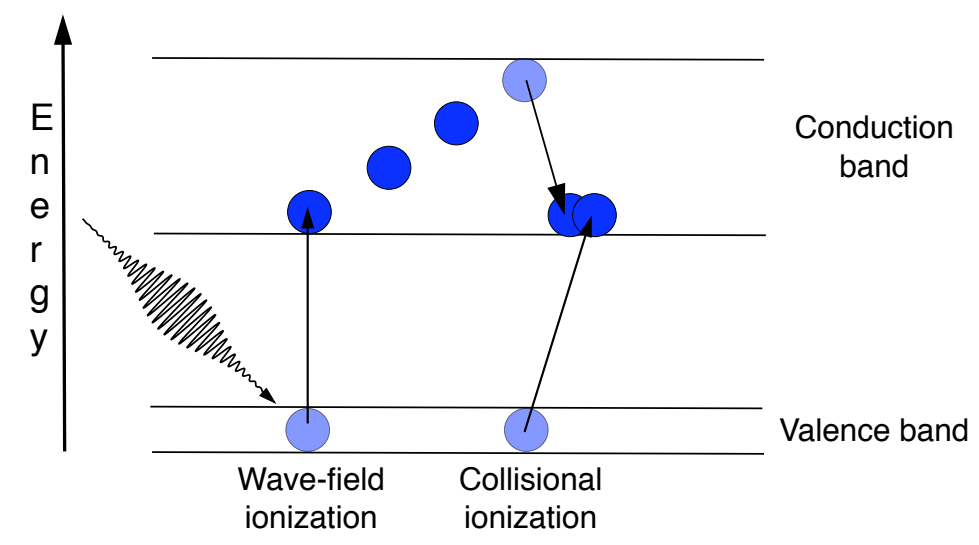

Figure 1: Different phenomena involved in generation of free electron gas.

In this paper, we consider a pulse (of wavelength $800 \mathrm{~nm}$ ) propagating in dielectric (silica). We assume that there is only one electron on the highest level of the valence band and that this level is not realimented. This is justified as strong ionization will not be considered in this work. The extension to more complicated materials is straightforward. Accounting for the whole valence band is the subject of a forthcoming work.

The laser pulse will evolve through Maxwell equations. For the free electrons, we use multiple rate equations (as in [18]) and a renormalization procedure to ensure energy conservation and render ohmic heating. The polarization of the material is given by a Bloch equation [6] or is directly computed from the number of neutrals [5]. 


\subsection{Light description}

To model the propagation of the laser in two and three dimensions, we use the fully time-dependent Maxwell equations. The laser pulse is described by the pair $(\mathbf{D}, \mathbf{H})$ evolving through Maxwell equations

$$
\left\{\begin{array}{l}
\mu_{0} \partial_{t} \mathbf{H}=-\nabla \times \mathbf{E} \\
\partial_{t} \mathbf{D}=\nabla \times \mathbf{H}-\mathbf{J} \\
\nabla \cdot \mathbf{D}=0 \\
\nabla \cdot \mathbf{H}=0
\end{array}\right.
$$

where we have $\mathbf{D}=\epsilon \mathbf{E}+\mathbf{P}$. The two relations $\nabla \cdot \mathbf{D}=0$ and $\nabla \cdot \mathbf{H}=0$ do not play any role in the sequel and are satisfied by the numerical schemes.

The interaction of light with matter occurs through the polarization $\mathbf{P}$ and current $\mathbf{J}$. The current $\mathbf{J}$ will be obtained from the densities and velocities of free electrons. We will see later on how to calculate these terms. We choose to present two different approaches for the computation of these terms in order to have a better control on the results.

\section{$2.2 \quad$ Free electrons}

Free electrons are described by their densities according to their energy. As in [18], we discretize the conduction band in $N_{p}$ levels whose mean energies (starting from the fundamental level $i=0$ ) are

$$
\mathcal{E}_{i}=\left(i+\frac{1}{2}\right) \hbar \omega_{L}, 0 \leq i \leq k,
$$

where $\hbar \omega_{L}$ is the photon energy of laser light and $k=\left[\frac{\mathcal{E}_{\text {crit }}}{\hbar \omega_{L}}+1\right]=N_{p}-1$, $\mathcal{E}_{\text {crit }}$ being the critical energy of ionization.

Remark 1 In fact, as the ohmic heating of the electrons is accounted for separately, there is no need to consider energy levels separated by the energy of a photon. The conduction band can be discretized differently as shown in Sec. 4.3

In the sequel, in our applications, we take $N_{p}=6$ (as we are considering fused silica but the extension to more energy levels is straightforward). The density of free electrons in the energy level $i$ at time $t$ and point $\mathbf{x}$ will be denoted by $n_{i}(t, \mathbf{x})$. In particular, the density of electrons above the critical energy for impact ionization is denoted by $n_{k}$.

For the time being, we consider three processes acting on the free electrons (see Fig. 1). The first one, multi-photon ionization depends on the laser pulse and the number of neutrals. The second one, collisional ionization, depends on the number of free electrons having reached the ionization 
energy and of the number of neutrals. Finally, the electrons also lose energy by radiation.

Collecting our assumptions, we can write them down as evolution equations. As in [18], we have to describe the evolution of each group density. The densities evolve through

$$
\left\{\begin{array}{l}
\partial_{t} n_{0}=\alpha_{\mathrm{ion}} N-\alpha_{\mathrm{des}} n_{0}+2 \alpha_{\mathrm{col}} \frac{N}{N_{0}} n_{k}, \\
\partial_{t} n_{i}=-\alpha_{\mathrm{des}} n_{i}, 0<i<k, \\
\partial_{t} n_{k}=-\alpha_{\mathrm{col}} \frac{N}{N_{0}} n_{k}-\alpha_{\mathrm{des}} n_{k},
\end{array}\right.
$$

where the rates $\alpha_{\mathrm{ion}}, \alpha_{\mathrm{des}}, \alpha_{\mathrm{col}}$ can be found in Sec. 2.7. The instantaneous number of neutrals is denoted by $N$. Let us also note that we have $N+$ $\sum_{i} n_{i}=N_{0}$ at every time. The evolution equation on $N$ is given in Eq. (6). The factor 2 in the first equation of system (3) comes from the fact that through collisional ionization, one electron in the $k$-th energy level ionizes one electron in the valence band: these two electrons are then in the lowest level of the conduction band.

Remark 2 There is no diffusion between groups, the cascading effect is not considered yet. These transitions will be accounted for later to ensure the conservation of energy in Sec. 2.5.

To each energy group, we associate a mean velocity denoted by $\mathbf{v}_{i}$ for the free electrons in the energy group $\mathcal{E}_{i}$. The evolutions of the different velocities of the free electrons are calculated using Drude's law [13]:

$$
\partial_{t} \mathbf{v}_{i}=\frac{Q_{e}}{m_{e}} \mathbf{E}-\nu_{e-}^{(i)} \mathbf{v}_{i}, 0 \leq i \leq k,
$$

where $Q_{e}$ is the charge of an electron, $m_{e}$ its mass and $\nu_{e}^{(i)}$ the collision rates containing electron-electron and neutral-electron collisions. Their expressions are obtained from

$$
\nu_{e}^{(i)}=\sigma_{\mathrm{eff}} \sqrt{\frac{2 \mathcal{E}_{i}}{m_{e}}} n_{i}
$$

as in [16].

\section{$2.3 \quad$ Neutrals and bound electrons}

We only consider one electron by atom. This electron is in the highest energy level. For each point $\mathbf{x}$ and time $t$, we denote by $N(t, \mathbf{x})$ the number of neutrals. According to our assumptions, this also corresponds to the number of bound electrons in the highest level of the valence band. 
The number of neutrals increases with the energy lost by radiation by the free electrons. This number decreases by multi-photon and collisional ionization. The total density $N$ of atoms obeys

$$
\partial_{t} N=\sum_{i=0}^{k} \alpha_{\text {des }} n_{i}-\alpha_{\text {ion }} N-\alpha_{\text {col }} n_{k} \frac{N}{N_{0}},
$$

$N_{0}$ being the number of neutrals at equilibrium without ionization. The transition rates $\alpha_{\text {ion }}, \alpha_{\text {des }}, \alpha_{\text {col }}$ are the same as those found in Eq. (3). Their expressions are given in Sec. 2.7. In this work, we do not consider that other species than neutrals could be produced by this recombination (e.g. negative ions).

To validate our rendering of the ionization process, we choose to use two different approaches for describing laser-matter interaction. The first one at the quantum-mechanical level uses the Bloch equation to describe the evolution of the material and the Maxwell equations for the light where the polarization term comes from the microscopic description. The second one is a model based on the Maxwell equations where the polarization term takes the dispersive effects into account.

Using the Bloch equation and the density matrix formulation $[7,15]$ : We describe the atoms according to their energy using the density matrix formalism. In this formalism, the material is statistically described at the quantum-mechanical level. Each molecule of the medium is considered as a quantum system with 2 discrete levels of energy.

The density matrix $\rho$ represents the various populations in each of the energy levels of the free Hamiltonian in its diagonal terms. The off-diagonal terms of the density matrix represent the quantum coherences of a set of atomic states. It was shown in [4], that this approach is adequate to describe a wide range of dispersive dielectrics.

Let us denote by $\omega_{j k}=\omega_{j}-\omega_{k}$ the difference between the two frequencies associated to levels $j$ and $k$. We shall also denote by $\mu$ the dipolar matrix of the material (depending on its microscopic structure) [7, 15]. The potential of interaction of the wave-field with the material is given by $V=-\mu \cdot \mathbf{E}$.

The density matrix $\rho$ satisfies

$$
\partial_{t} \rho_{\ell \ell}=\frac{i}{\hbar}[V, \rho]_{\ell \ell},
$$

(where we remind that for two operators $A$ and $B,[A, B]=A B-B A$ ) for the population terms and the following equations for the coherences.

$$
\partial_{t} \rho_{j k}=-\imath \omega_{j k} \rho_{j k}+\frac{\imath}{\hbar}[V, \rho]_{j k}, j \neq k .
$$


The polarization is given by

$$
\mathbf{P}_{a}=N \operatorname{tr}(\mu \rho),
$$

hence its time derivative is computed through

$$
\partial_{t} \mathbf{P}_{a}=\operatorname{tr}(\mu \rho) \partial_{t} N+N \operatorname{tr}\left(\mu \partial_{t} \rho\right) .
$$

This approach will be useful for introducing the influence of the lower level electrons.

Using a nonlinear polarization in the Maxwell equations: The polarization in (1) is obtained from

$$
\mathbf{P}=\frac{N}{N_{0}}\left(\mathbf{P}_{L}+\mathbf{P}_{N L}\right)
$$

where the linear $\mathbf{P}_{L}$ and nonlinear $\mathbf{P}_{N L}$ terms are computed as in [5]. The number of neutrals without the laser pulse is denoted by $N_{0}$.

This approach is useful to account for a Kerr-type nonlinearity.

\subsection{Interaction terms}

The currents involved in Maxwell equations (1) are decomposed into $\mathbf{J}=$ $\mathbf{J}_{e}+\mathbf{J}_{\mathrm{mpi}}$. The first one is due to the movement of free electrons and the ionization is responsible for the second one.

The first current is obtained from the expression

$$
\mathbf{J}_{e}=\sum_{i=0}^{i_{0}} Q_{e} n_{i} \mathbf{v}_{i}
$$

Then we have to compute the current due to photonic ionization. Its expression is given by

$$
\mathbf{J}_{\mathrm{mpi}}=\frac{\alpha_{\text {ion }} W_{\text {ion }} N}{\mathbf{E}^{2}} \mathbf{E},
$$

where for silica $W_{\text {ion }}=9\left|Q_{e}\right|, Q_{e}$ being the charge of an electron, $\mathbf{E} \cdot \mathbf{J}_{\mathrm{mpi}}=$ $\alpha_{\text {ion }} W_{\text {ion }}$ is the ionization energy [16,20]. If the electric field is vanishing, we take $\mathbf{J}_{\mathrm{mpi}}=0$. The expression of $\alpha_{\mathrm{ion}}$ is found in Sec. 2.7 and depends on the intensity of the incoming wave-field.

\subsection{Diffusion between energy groups: ohmic heating}

At this stage, we have not taken into account the effect of the acceleration of electrons by the laser and the transition it generates between energy groups. In [18], this is achieved directly in the rate equations. However, 
the corresponding probability or avalanche coefficient is difficult to recover experimentally and is generally intuitively estimated $[2,20]$. We choose another approach to render these physical effects. Equations (3) do not contain cascading terms. Free electrons are heated by the laser pulse and their energy increases. We have assumed that there are $n_{i}$ electrons with mean energy $\mathcal{E}_{i}$ (or with an energy between $i \hbar \omega_{L}$ and $(i+1) \hbar \omega_{L}$ ). If these electrons are heated they may leave this group to a group of electrons with higher energy.

We choose to compute the number of these electrons leaving the current group and add them to the appropriate group. Yet, if we add these electrons to a different group, the quantity of movement of the first group changes. Then, we also have to adjust the velocities in each group.

Initially, we consider that all electrons are in the lowest level $n_{0}$. We will denote by $i_{0}(t, \mathbf{x})$, the highest populated energy level at the point $\mathbf{x}$ and time $t$. We have $i_{0}(0, \mathbf{x})=0$ and $i_{0}(t, \mathbf{x}) \leq k$.

In the sequel, we use the notation $\omega_{c}=\frac{W_{\text {ion }}}{k \hbar}$, where $W_{\text {ion }}$ is the energy of ionization of the material.

Renormalization of densities: The laser heating makes electrons gain energy and thus they may change from one energy level to another. We will denote by $\mathcal{E}_{i}^{*}$ the energy obtained from $\mathcal{E}_{i}$. If $\mathcal{E}_{i}^{*}>\mathcal{E}$, there will be a flux of electrons from the energy group $\mathcal{E}_{i}$ to $\mathcal{E}_{i+1}$.

The principle of the projection is shown in Fig. 2. We consider that the electrons are found uniformly in each group. So the transition $\mathcal{E}_{0} \rightarrow \mathcal{E}_{0}^{*}$ gives rise to a flux

$$
\delta n_{0}=\frac{\mathcal{E}_{0}^{*}-\mathcal{E}_{0}}{\mathcal{E}_{0}^{*}-0} n_{0}
$$

corresponding to the second gray rectangle in Fig. 2 and for the higher groups we have

$$
\delta n_{j}=\frac{\mathcal{E}_{j}^{*}-\mathcal{E}_{j}}{\mathcal{E}_{j}^{*}-\mathcal{E}_{j-1}^{*}} n_{j} .
$$

We have

$$
\begin{aligned}
\mathcal{E}_{j}^{*}-\mathcal{E}_{j-1}^{*} & =\mathcal{E}_{j}^{*}-\mathcal{E}_{j}+\mathcal{E}_{j}-\mathcal{E}_{j-1}+\mathcal{E}_{j_{1}}-\mathcal{E}_{j-1}^{*}, \\
& =\Delta \mathcal{E}_{j}+\hbar \omega_{c}+\Delta \mathcal{E}_{j-1}
\end{aligned}
$$

where $\Delta \mathcal{E}_{j}=\mathcal{E}_{j}^{*}-\mathcal{E}_{j}$.

To compute the variation of energy, we use

$$
\partial_{t} \mathcal{E}_{i}=Q_{e} \mathbf{v}_{i} \cdot \mathbf{E},
$$

which gives the flux between two groups

$$
\Delta \mathcal{E}_{i} \equiv \mathcal{E}_{i}^{*}-\mathcal{E}_{i}=\delta t Q_{e} \mathbf{E} \cdot\left(\frac{\mathbf{v}_{i}+\mathbf{v}_{i-1}}{2}\right),
$$




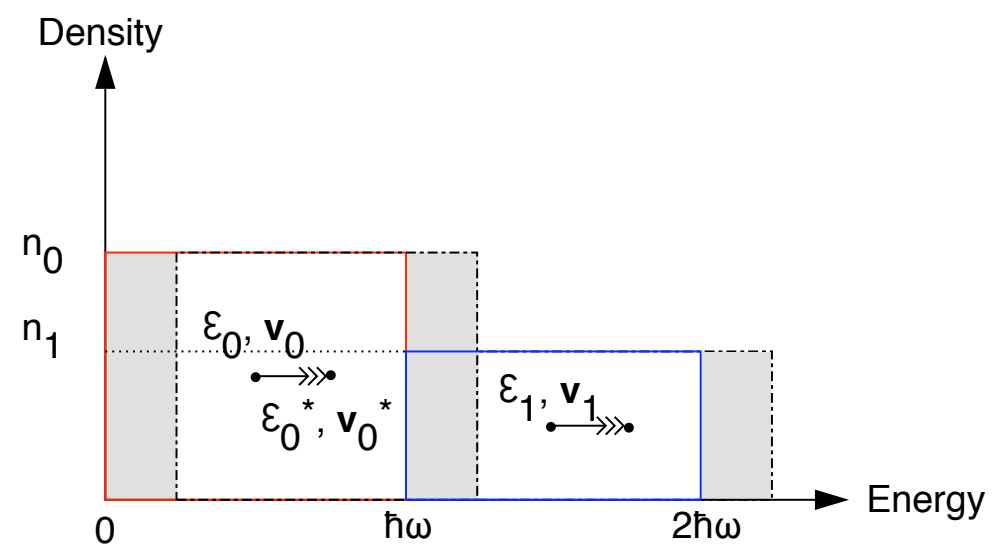

Figure 2: Principle of the computation of the new densities to take heating into account. The red and blue rectangles represents the two initial groups corresponding to energies $\mathcal{E}_{0}$ and $\mathcal{E}_{1}$. After heating, these two groups are shifted and have to be projected to compute the new densities of each energy level. We assume that the electrons are uniformly distributed in each group. 
where we have approximated the velocity of the interface between groups $i-1$ and $i$ by $\frac{\mathbf{v}_{i}+\mathbf{v}_{i-1}}{2}$.

We compute

$$
\left\{\begin{aligned}
\tilde{n}_{0} & =n_{0}-\delta n_{0} \\
\tilde{n}_{i} & =n_{i}+\delta n_{i-1}-\delta n_{i}, 1 \leq i \leq i_{0}-1 \\
\tilde{n}_{i_{0}} & =n_{i_{0}}+\delta n_{i_{0}-1} .
\end{aligned}\right.
$$

Remark 3 It shall be noted that this process does not change the total number of free electrons.

Renormalization of velocities: The total momentum per group must also be computed as we have changed the electron densities. The momentum of the energy group $\mathcal{E}_{i}$ is given by

$$
\mathbf{p}_{i}=m_{e} n_{i} \mathbf{v}_{i} .
$$

The quantity of movement leaving the first group at the interface is given by

$$
\delta \mathbf{p}_{0}=-m_{e} \delta n_{0}\left(\frac{\mathbf{v}_{0}+\mathbf{v}_{1}}{2}\right),
$$

and for the other levels we obtain

$$
\delta \mathbf{p}_{i}=m_{e}\left(\delta n_{i-1} \frac{\mathbf{v}_{i-1}+\mathbf{v}_{i}}{2}-\delta n_{i} \frac{\mathbf{v}_{i}+\mathbf{v}_{i+1}}{2}\right) .
$$

Then we compute the adjusted velocities with

$$
\tilde{\mathbf{v}}_{i}=\frac{m_{e} n_{i} \mathbf{v}_{i}+\delta \mathbf{p}_{i}}{m_{e} \tilde{n}_{i}}, 0 \leq i \leq i_{0} .
$$

Renormalization of last group: As electrons are heated, their energy can exceed the threshold $\mathcal{E}_{i_{0}}$. In this case, we need to add an additional energy level (for instance, with $i_{0}=1$, we have to add an energy level to account for free electrons in the right-most gray rectangle of Fig. 2). For this matter, we use the conservation of energy.

Let us denote by $U_{i}$ the total energy of the group $i$. We have $U_{i}=$ $\left(i+\frac{1}{2}\right) \hbar \omega_{c} n_{i}$ for $0 \leq i<i_{0}$, the energy in the last group ensures the conservation of the total energy.

First, we compute the total energy in the material

$$
S_{o}=\sum_{i=0}^{i_{0}} U_{i}, S=\sum_{i=0}^{i_{0}-1}\left(i+\frac{1}{2}\right) \hbar \omega_{c} n_{i} .
$$


The total energy created by ohmic heating during $d t$ is given by

$$
S_{c}=\sum_{i=0}^{i_{0}} Q_{e} n_{i}\left(\mathbf{v}_{i} \cdot \mathbf{E}\right) d t
$$

and the energy due to radiation is

$$
S_{r}=\sum_{i=0}^{i_{0}-1}\left(i+\frac{1}{2}\right) \hbar \omega_{c} n_{e}\left(1-e^{-\nu_{\mathrm{des}} d t}\right)+U\left(i_{0}\right)\left(1-e^{-\nu_{\mathrm{des}} d t}\right),
$$

where we have used Eq. (3) to evaluate the energy lost by radiation during a time $d t$.

The energy of the last level is given by

$$
U\left(i_{0}\right)=S_{o}-S-S_{r}+S_{c}+\delta n_{k}\left(\hbar \omega_{c}-\frac{U(k)}{n_{k}}\right) .
$$

We shall now decide if there is a need to add another energy level (assuming that $i_{0}<6$ ). If $U\left(i_{0}\right)>n_{i_{0}}\left(i_{0}+\frac{1}{2}\right) \hbar \omega_{c}$, we have to create a new level. To ensure that the last level is not almost empty, we will only add a level if $U\left(i_{0}\right)>n_{i_{0}}\left(i_{0}+1\right) \hbar \omega_{c}$.

The energy per electron is given by $\frac{U\left(i_{0}\right)}{n_{i_{0}}}$. Then assuming that the electrons are uniformly distributed we get

$$
\delta n\left(i_{0}\right)=n_{i_{0}} \frac{\frac{U\left(i_{0}\right)}{n_{i_{0}}}-\left(2 i_{0}+1\right) \hbar \omega_{c}}{\frac{U\left(i_{0}\right)}{n_{i_{0}}}-i_{0} \hbar \omega_{c}},
$$

and we set the new values of the densities $n^{*}\left(i_{0}\right), n^{*}\left(i_{0}+1\right)$ and energies $U^{*}\left(i_{0}\right), U^{*}\left(i_{0}+1\right)$ :

$$
\begin{aligned}
& n_{i_{0}}^{*}=n_{i_{0}}-\delta n\left(i_{0}\right) ; n_{i_{0}+1}^{*}=\delta n\left(i_{0}\right) \\
& U^{*}\left(i_{0}+1\right)=U\left(i_{0}\right)-\left(i_{0}+\frac{1}{2} \hbar \omega_{c}\right) n_{i_{0}}^{*} ; U^{*}\left(i_{0}\right)=\left(i_{0}+\frac{1}{2} \hbar \omega_{c}\right) n_{i_{0}}^{*}
\end{aligned}
$$

Finally, we have to compute the adequate velocities. For that matter, we use the same method as in the previous paragraph. We ensure that we have the conservation of momentum. The momentum of the group we have just added must correspond to the initial momentum of the group $i_{0}$ minus the momentum of the updated group $i_{0}$.

For the sake of simplicity, let us assume that $i_{0}=0$. We denote by $\overline{\mathbf{v}}$ the velocity of the interface between groups 0 and 1. As in Eq. (19), the momentum leaving the first group is given by

$$
\delta \mathbf{p}_{0}=-m_{e} \delta n_{0} \overline{\mathbf{v}},
$$


but this time we have to compute the velocity of the interface. We choose to use a linear approximation i.e. we take $\overline{\mathbf{v}}=2 \mathbf{v}_{0}$. We have conservation of the momentum, which (using the same notations as before) leads to

$$
m_{e} n_{0}^{*} \mathbf{v}_{0}^{*}=m_{e} n_{0} \mathbf{v}_{0}+\delta \mathbf{p}_{0} .
$$

We replace $\delta \mathbf{p}_{0}$ and $\overline{\mathbf{v}}$ by their expressions and we obtain

$$
\mathbf{v}_{0}^{*}=\left(1-\frac{\delta n_{0}}{n_{0}^{*}}\right) \mathbf{v}_{0} .
$$

Finally, we take

$$
\mathbf{v}_{1}^{*}=\overline{\mathbf{v}} \text {. }
$$

Remark 4 One can also use an approximation of higher order.

\subsection{Adding a relaxation process toward thermal equi- librium}

We now assume that the electronic densities are relaxing toward a Maxwellian distribution [20]. That is that we assume a Maxwellian distribution for the electron velocity i.e.

$$
F(v)=\frac{1}{\sqrt{\pi} v_{t h}} \exp \left(-\frac{v^{2}}{v_{t h}}\right)
$$

To obtain the corresponding energy distribution function $f_{e}$, we take

$$
W=W_{t h} \frac{v^{2}}{v_{t h}^{2}},
$$

where $W_{t h}=\frac{1}{2} m v_{t h}^{2}=\frac{3}{2} k_{B} T_{e}\left(T_{e}\right.$ being the electronic temperature). This gives

$$
f_{e}(W)=\frac{1}{2 \sqrt{\pi W_{t h} W}} \exp \left(-\frac{W}{W_{t h}}\right) .
$$

The equations on electron densities become

$$
\left\{\begin{array}{c}
\partial_{t} n_{0}=\alpha_{\text {ion }} N-\alpha_{\text {des }} n_{0}+2 \alpha_{\text {col }} \frac{N}{N_{0}} n_{k}-\frac{1}{\tau_{m}}\left(n_{0}-n_{0}^{e q}\right), \\
\partial_{t} n_{i}=-\alpha_{\text {des }} n_{i}-\frac{1}{\tau_{m}}\left(n_{i}-n_{i}^{e q}\right), 0<i<k, \\
\partial_{t} n_{k}=-\alpha_{\text {col }} \frac{N}{N_{0}} n_{k}-\alpha_{\text {des }} n_{k}-\frac{1}{\tau_{m}}\left(n_{k}-n_{k}^{e q}\right),
\end{array}\right.
$$

where we have denoted by $\tau_{m}$ the relaxation time toward equilibrium (identical for each level). 
We shall now compute the densities at equilibrium. We have

$$
\begin{aligned}
n_{i}^{e q} & =\frac{\sum_{j} n_{j}}{k T} \int_{i \hbar \omega}^{(i+1) \hbar \omega} \exp \left(-\frac{W}{k T}\right) d W, 0 \leq i<k \\
& =\left(\sum_{j} n_{j}\right)\left(1-\exp \left(-\frac{\hbar \omega}{k T}\right)\right) \exp \left(-\frac{i \hbar \omega}{k T}\right), 0 \leq i<k,
\end{aligned}
$$

and

$$
n_{k}^{e q}=\left(\sum_{j} n_{j}\right) \exp \left(-\frac{k \hbar \omega}{k T}\right) .
$$

Let us note that we have $\sum_{j} n_{j}^{e q}=\sum_{j} n_{j}$.

Remark 5 In our experiments, most of the time the highest level is not populated. Therefore this relaxation process toward equilibrium will accelerate the ionization.

\subsection{Probabilities}

We have to determine the probabilities associated to each of the three processes involved. We have taken the expression found in [16]. For reader's convenience, we recall these expressions shortly.

For the probability of collision, we have used the expressions from [16], namely

$$
\alpha_{\mathrm{col}}=\frac{2 \alpha_{\mathrm{col}}^{0}}{\sqrt{\pi}} \frac{N}{N_{0}}\left(\left(\frac{15}{4} \beta^{\frac{3}{2}}-\frac{1}{2} \sqrt{\beta}\right) e^{-\frac{1}{\beta}}+\sqrt{\frac{\pi}{2}}\left(\frac{15}{4} \beta^{2}-3 \beta+1\right) \operatorname{erfc}\left(\frac{1}{\sqrt{\beta}}\right)\right)
$$

where $\beta=\frac{W_{\text {th }}}{W_{\text {ion }}}, N_{0}=2 \times 10^{28} \mathrm{~m}^{-3}$ and $\alpha_{\text {col }}^{0}=1.5 \times 10^{17}$. We have denoted by erfc the function $1-\frac{2}{\sqrt{\pi}} \int_{0}^{x} \exp \left(-t^{2}\right) d t$.

The probability of radiation is also a constant value:

$$
\alpha_{\mathrm{des}}=\frac{1}{\tau_{\text {trap }}}=\left(1.5 \times 10^{-13}\right)^{-1} \mathrm{~s}^{-1},
$$

see [16] for details. The influence of this phenomenon is probably limited with ultrashort pulses.

The probability of ionization depends on the intensity of the incoming wave-field.

$$
\alpha_{\text {ion }}=\frac{\sigma_{6}}{N_{0}}\left(10^{-12} \mathbf{E} \times \mathbf{H}\right)^{6},
$$

as in [16] we took $\sigma_{6}=2 \times 10^{7} \mathrm{~m}^{-3} \mathrm{~s}^{-1}\left(\mathrm{TW} / \mathrm{m}^{2}\right)^{-6}$ and $N_{0}=2 \times 10^{28} \mathrm{~m}^{-3}$. 


\section{$3 \quad$ Numerical method}

\subsection{Discretization of the Maxwell equations}

For discretizing Maxwell equations, we use a finite-difference scheme based on Yee's work [23]. The physical variables are computed using the scheme shown in Figure 3. For a detailed presentation of this scheme, one can see $[6]$.

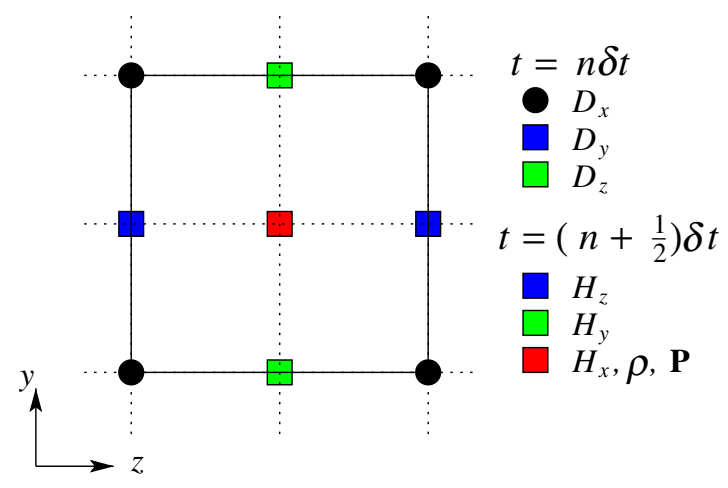

Figure 3: Scheme used to discretize the physical variables.

The discretization of the model based on nonlinear Maxwell equations is described in [5].

\subsection{Computation of free electrons densities}

The computation of the free electron densities given by (3) is decomposed in two parts using a splitting method. The first one is completely linear

$$
\left\{\begin{array}{l}
\partial_{t} n_{0}=\alpha_{\text {ion }} N-\alpha_{\text {des }} n_{0}-\frac{1}{\tau_{m}}\left(n_{0}-n_{0}^{e q}\right), \\
\partial_{t} n_{i}=-\alpha_{\text {des }} n_{i}-\frac{1}{\tau_{m}}\left(n_{i}-n_{i}^{e q}\right), 0<i<k, \\
\partial_{t} n_{k}=-\alpha_{\text {des }} n_{k}-\frac{1}{\tau_{m}}\left(n_{k}-n_{k}^{e q}\right),
\end{array}\right.
$$

and the second one is nonlinear but only involves the lowest and highest energy level:

$$
\left\{\begin{aligned}
\partial_{t} n_{0} & =2 \alpha_{\operatorname{col}} \frac{N}{N_{0}} n_{k} \\
\partial_{t} n_{k} & =-\alpha_{\operatorname{col}} \frac{N}{N_{0}} n_{k} \\
N & =N_{0}-n_{0}-\sum_{i=1}^{k-1} n_{i}-n_{k}
\end{aligned}\right.
$$




\subsubsection{Resolution of (39)}

As the system corresponding to (39) is linear, we will use an exponential scheme. Hence we will compute

$n_{j}^{n+1}=n_{j}^{n} e^{-\left(\alpha_{\operatorname{des}}(j)+\frac{1}{\tau_{m}}\right) d t}+\frac{n_{i}^{e q}}{\tau_{m} \alpha_{\operatorname{des}}(j)+1}\left(1-e^{-\left(\alpha_{\operatorname{des}}(j)+\frac{1}{\tau_{m}}\right) d t}\right), 1 \leq j \leq k$,

and for the lowest level we have

$$
n_{0}^{n+1}=n_{0}^{n} e^{-\left(\alpha_{\mathrm{des}}(0)+\frac{1}{\tau_{m}}\right) d t}+\frac{\alpha_{\mathrm{ion}} N^{n+\frac{1}{2}}+\frac{1}{\tau_{m}} n_{0}^{e q}}{\alpha_{\mathrm{des}}(0)+\frac{1}{\tau_{m}}}\left(1-e^{-\left(\alpha_{\mathrm{des}}(0)+\frac{1}{\tau_{m}}\right) d t}\right) .
$$

\subsubsection{Resolution of (40)}

We have to compute the densities from (40) (i.e. we are splitting the equations on $n_{0}$ and $n_{k}$ in time). Summing the two first equations of (40), we get $\partial_{t}\left(n_{0}+2 n_{k}\right)=0$, hence $n_{0}^{n+1}+2 n_{k}^{n+1}=n_{0}^{n}+2 n_{k}^{n}$.

We compute

$$
n_{k}^{n+1}=e^{-\frac{\alpha_{\mathrm{col}} N^{n+\frac{1}{2}}}{N_{0}}} d t n_{k}^{n}
$$

and

$$
n_{0}^{n+1}=n_{0}^{n}+2\left(n_{k}^{n}-n_{k}^{n+1}\right)
$$

\subsection{Computation of the electrons velocities}

One has to compute the velocities with equations (4). For this purpose, we use an exponential scheme yielding

$$
\mathbf{v}_{i}^{n+1}=e^{-\nu_{e} d t} \mathbf{v}_{i}^{n}+\frac{Q_{e}}{m_{e} \nu_{e}}\left(1-e^{-\nu_{e} d t}\right) \mathbf{E},
$$

where this equation is taken component by component.

\section{Numerical results}

The two approaches described in Sec. 2.3 yield identical results. Both will be used in this section. 


\subsection{Propagation of an ultrashort pulse in fused silica}

In this experiment, we compute the propagation of an ultrashort pulse in fused silica. The intensity of the $50 \mathrm{fs}$ pulse $(\lambda=800 \mathrm{~nm})$ is $1.5 \times 10^{10} \mathrm{~V} / \mathrm{m}$. We have considered $3 \mu \mathrm{m}$ of silica.

The initial density of atoms is $N_{n}^{0}=2 \times 10^{28} \mathrm{~m}^{-3}$, and we have $n_{e}^{0}=$ $10^{10} \mathrm{~m}^{-3}$ free electrons at each point of the dielectric.

We have taken 100 points per wavelength in the direction of propagation and 150 points in the transverse direction. The time step is computed to ensure the CFL condition.

The initial wave-field is given by $\mathbf{E}(t, y)=E_{\max } \sin \left(\frac{\pi t}{\tau}\right) \cos \left(\omega_{0}(t-\right.$ $\left.\left.\frac{\tau}{2}\right)\right) e^{-\frac{1}{2}\left(\frac{y}{w_{y}}\right)^{2}} H(\tau-t)$, where $w_{y}=0.5 \mu \mathrm{m}, E_{\max }=1.5 \times 10^{10} \mathrm{~V} / \mathrm{m}, \tau=50 \mathrm{fs}$. Thus the input laser intensity is roughly $8.8 \times 10^{13} \mathrm{~W} / \mathrm{cm}^{2}$ and the peak power $689 \mathrm{~kW}$.

The amplitude of the electric field $E_{y}$ after 35 fs is shown in Fig. 4.

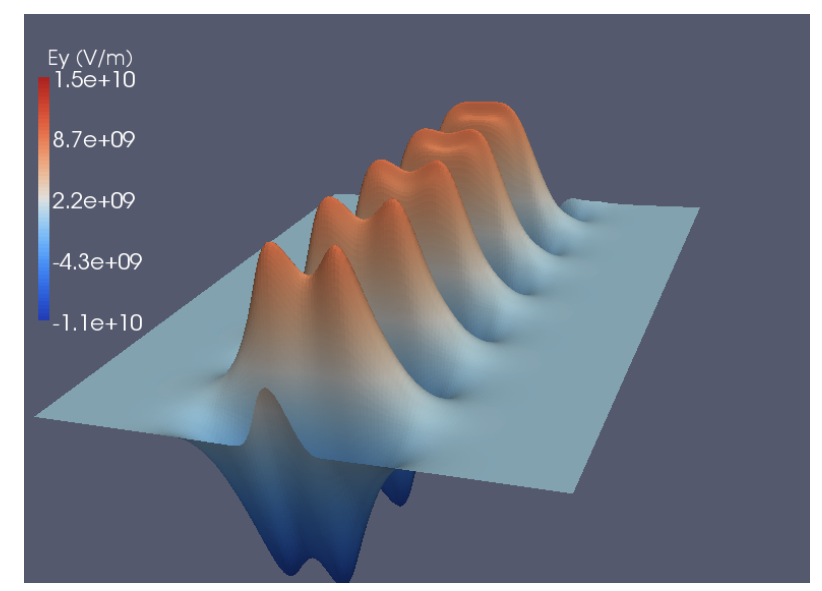

Figure 4: Amplitude of the electric field $E_{y}$ after 35 fs of propagation. The pulse propagates from bottom left to top right.

The absorption at the center of the pulse is clearly visible. To underline the nonlinear effects, the spectrum of this field is shown in Fig. 5. Harmonic generation is clearly observed and only odd harmonics appear as expected. The ionization process is a source of nonlinearities, the nonlinear index of the material is also taken into account.

We now show the evolutions of the different populations of electrons at the front of the dielectric in the center of the pulse. These evolutions are shown in Fig. 6. The medium is almost completely ionized by the laser 


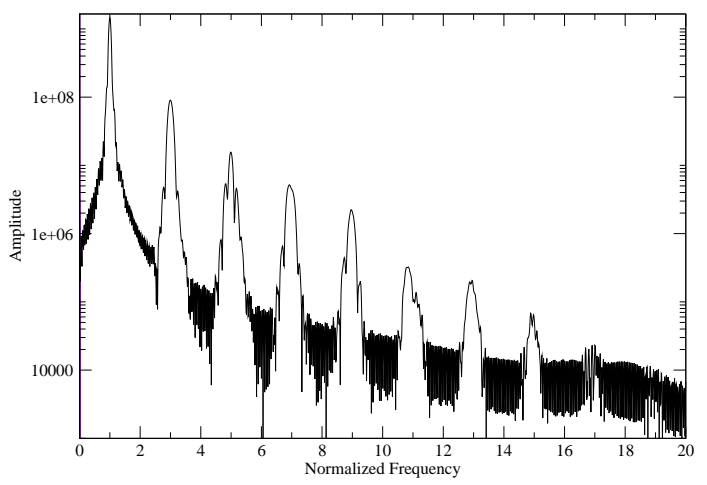

Figure 5: Fourier transform of the wave-field in the $y$ polarization in the center of the material after $35 \mathrm{fs}$ of propagation. The amplitude is shown in arbitrary units in a log scale. The frequency scale is normalized by the frequency of the incoming wave.

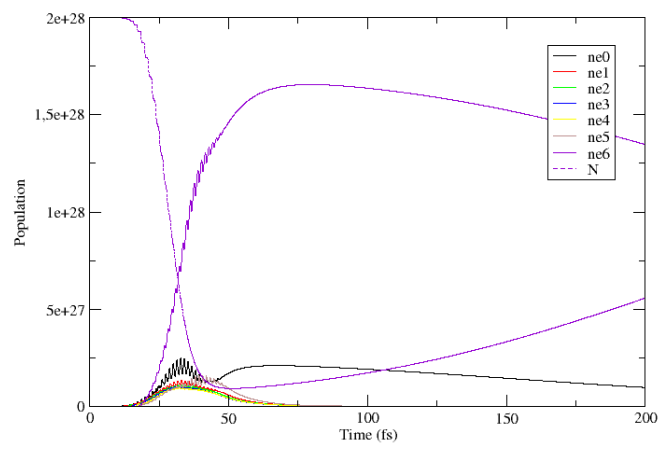

Figure 6: Evolution of the populations of electrons at the center of the pulse at the front of the material over time. The plain lines denote the different populations of free electrons corresponding to the seven energy levels $(0, \ldots 6)$ and the dotted line represents the population of neutrals. 
beam and a large number of electrons reach the highest energy level of the conduction band.

\subsection{Influence of Maxwellian relaxation}

In this section, we study the effect of the relaxation toward a Maxwellian equilibrium. This process is added in our model in section 2.6.

We use the same experimental setup than in the previous experiment with two different values for the relaxation time $\left(\tau_{m}\right.$ in Eq. (34)). Let us note that the value $\tau_{m}=1 \mathrm{fs}$ is shorter than one optical cycle. We choose such a small value to observe the effect of the relaxation process.

The result of the run is shown in Fig. 7.

As initially higher levels are not populated, the relaxation process increases ionization. In order to be able to observe the effect of this relaxation, we had to take a very short relaxation time $\left(\tau_{m}=1 \mathrm{fs}\right)$ which is probably not realistic. With a more reasonable value, there is almost no difference with the evolution we observe without this Maxwellian relaxation (in Fig. 6).

The effect of this relaxation on the number of free electrons is quite small, we believe it can be safely neglected. The electrons are not very hot and the relaxation time is thus too long for this process to be influent.

\subsection{Influence of the discretization of the conduction band}

Contrary to other models like [18], the one-photon absorption by free electrons is not directly accounted in the equations (3). These energy levels do not have to be separated exactly by the energy of one photon. Thus we can discretize the conduction band as we wish as long as the difference between the first and the last level is conserved.

In this experiment, we wish to study the influence of this discretization on the ionization. For instance, we can see if a finer discretization is needed to better account for the cascading effect. We have run the same experiment with three different discretizations of the conduction band. One has 5 levels, the second one is the same we have used before (7 levels) and last one has 2 additional levels.

It shall also be noted that, of course, refining the discretization increases the computation time and that finer discretizations may require smaller time steps (however it is not the case in this test).

We have shown the evolution of the number of neutrals and the number of free electrons in the lowest energy level in Fig. 8.

There is almost no difference on the number of neutrals (or the total number of free electrons). The population of electrons in the level $\mathcal{E}_{0}$ is a 

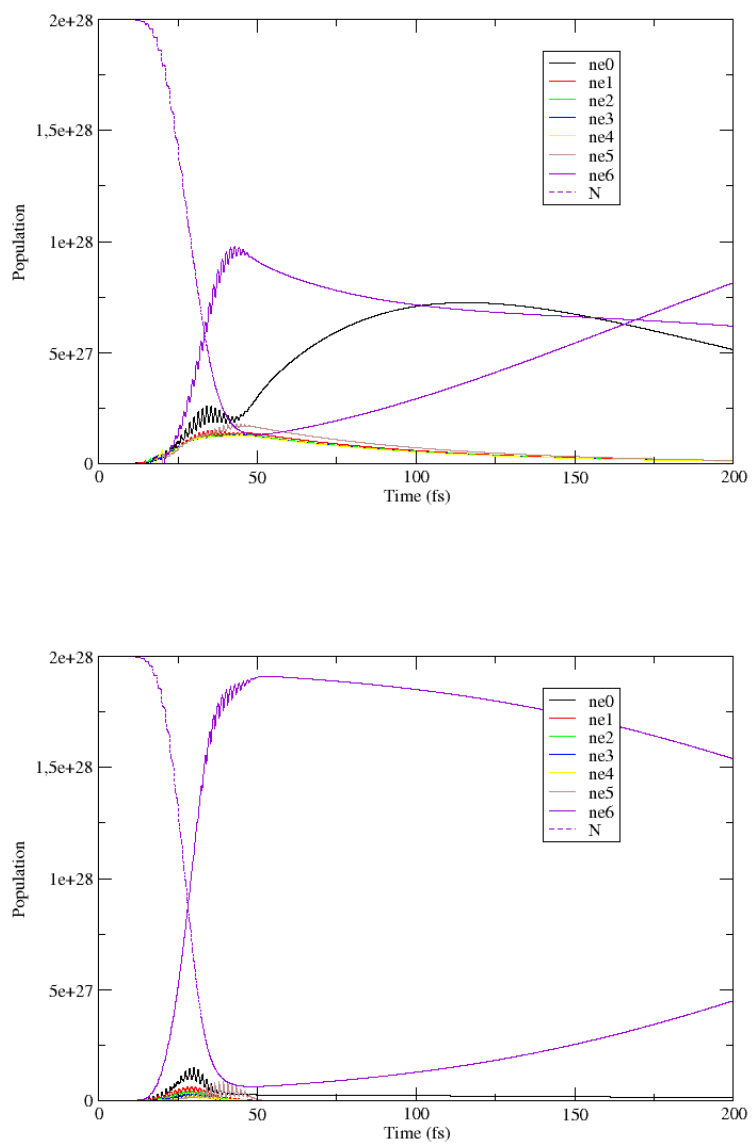

Figure 7: Electron populations over time in the setup of Sec. 4.1 with $\tau_{m}=100 \mathrm{fs}$ (first picture) and $\tau_{m}=1 \mathrm{fs}$ (second picture) in the relaxation process added in Sec. 2.6. 


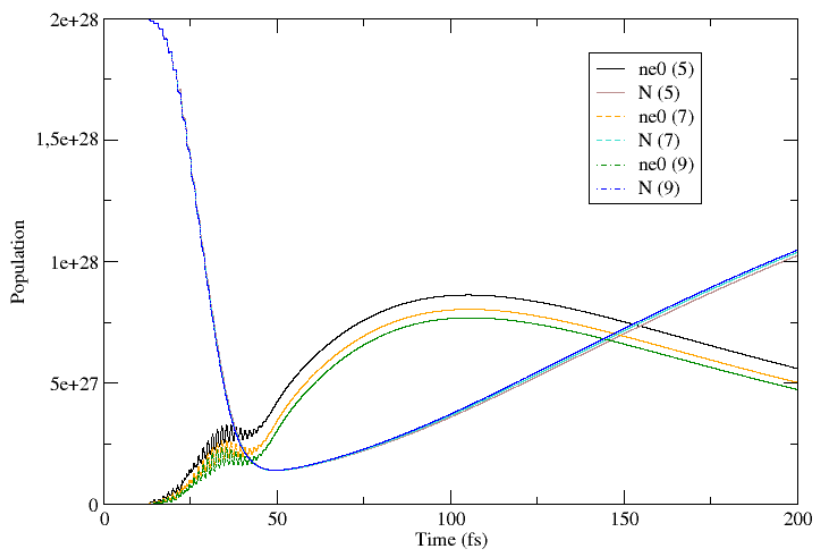

Figure 8: Electron populations over time in the setup of Sec. 4.1 with one finer (9 points), one standard ( 7 points) and one coarser discretization ( 5 points) of the conduction band. 
little smaller with the coarser discretization. As the second level is closer to the first one with a finer grid, it is probably easier to reach for an electron. Hence, the population in the lowest level is smaller.

\subsection{Comparison with the model [16]}

In this experiment, we compare our model with the model described in [16]. This model only uses one group for the free electrons and assumes that these electrons are at thermal equilibrium.

In Fig. 9, we have plotted the evolution of energy fluxes of our model and the model [16]. Computations were performed in $1 \mathrm{D}$ and $2 \mathrm{D}$ (the picture represents the evolution of the flux inside the dielectric as a function of the depth).

The same experiment is also run in 3D. The result is shown in Fig. 10.

In Fig. 11, we have plotted the total number of free electrons for three different points in the dielectric over time for the two models. These points are uniformely distributed at the center of the material in the $3 \mu \mathrm{m}$ of the width.

Finally, we have plotted, in Fig. 12 and 13, the absorbed energy. The pulse propagates from the back of the figure in the $z$-direction. The first picture represents the absorbed energy (in J) for the temperature model [16]. We can observe that the absorption occurs mainly at the surface of the dielectric. The width of the material is as before $3 \mu \mathrm{m}$.

In Fig. 13, we show the result of the same experiment with the multigroup model. As before, one can observe that the material is more ionized with this model than with the temperature model. The largest contour shown in this picture represents the maximal value of absorbed energy obtained with the temperature model (more precisely this contour corresponds to $E_{\text {abs }}=2.6 \times 10^{10} \mathrm{~J}$ ).

Even if the results look similar, with the model [16], the material is less ionized than with our model. Without additional information from experiments, it is hard to determine which model is the most accurate. However, let us note that our model is obtained from less restrictive assumptions than [16]. Indeed, the electronic distribution observed with the multi-group approach does not correspond to a thermic equilibrium.

It is nevertheless interesting to note that because the absorption occurs more in the bulk for the temperature model, the reflection of the pulse is less effective. This explains why the energy inside the dielectric is greater for the temperature model although the pulses are the same. This effect is more visible in $1 \mathrm{D}$ than in $2 \mathrm{D}$ or $3 \mathrm{D}$. Actually, the reflection in $3 \mathrm{D}$ is quite negligible. 

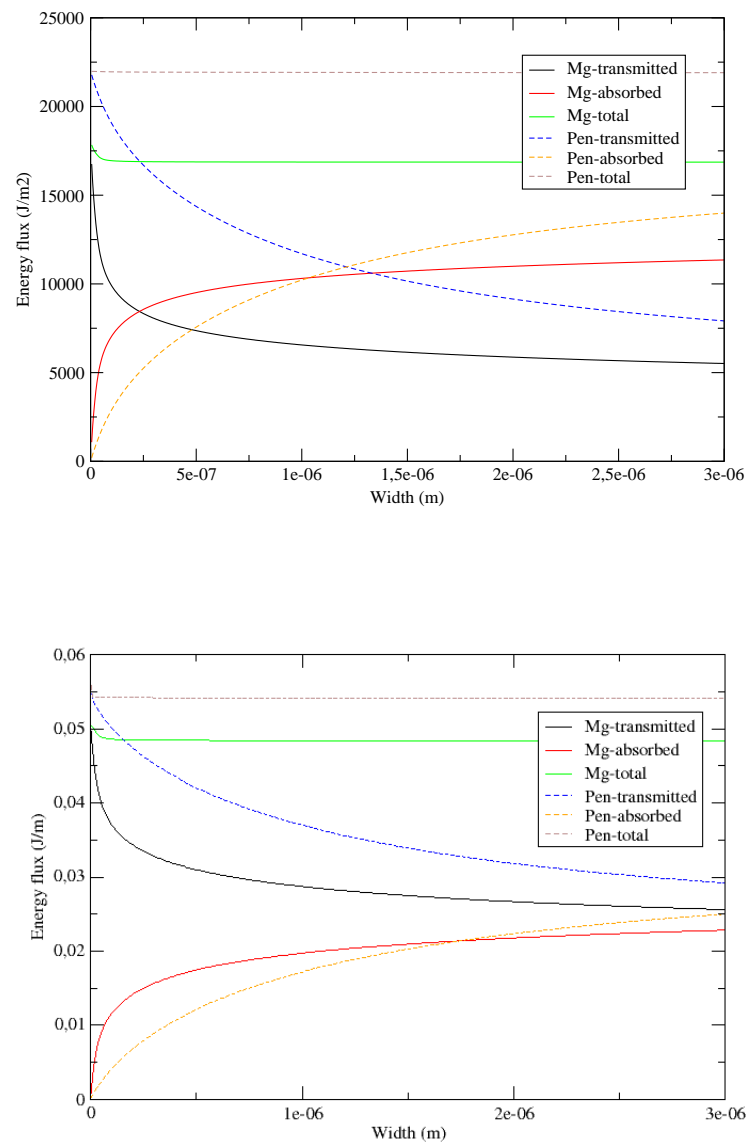

Figure 9: Energy fluxes in $\mathrm{J} / \mathrm{m}^{2}$ (1D, first picture), $\mathrm{J} / \mathrm{m}(2 \mathrm{D}$, second picture) over width $(\mathrm{m})$ for our model (i.e. quantities prefixed with "Mg") and the model [16] (quantities prefixed with "Pen") in 1D and 2D (in the center of the dielectric). We plot for each approach the absorbed, transmitted and total energy. 


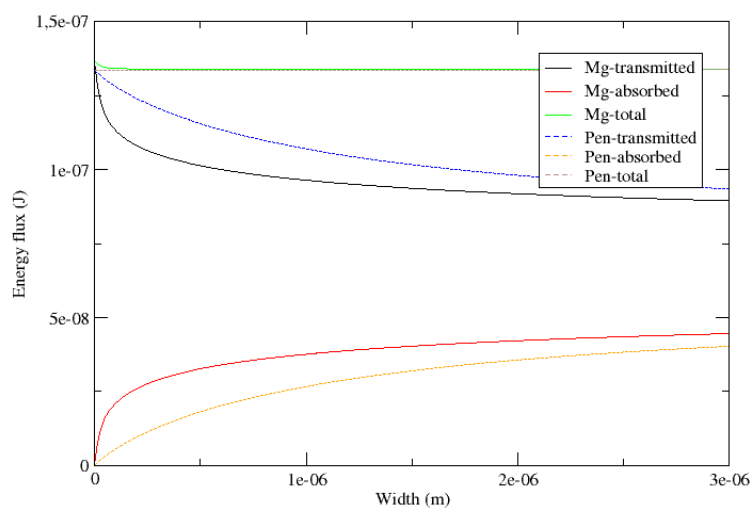

Figure 10: Energy $(\mathrm{J})$ over width $(\mathrm{m})$ obtained from our model (quantities prefixed with "Mg") and the model [16] (quantities prefixed with "Pen") in 3D. We plot for each approach the absorbed, transmitted and total energy.

\subsection{Experimental comparison}

In this section, we will use the experimental results presented in [14] to study the accuracy of our model.

The detailed description of the experimental setup is given in [14]. The authors analyze the phase shift of probe laser pulse propagating in a sample of $\mathrm{SiO}_{2}$ by observing the pattern resulting from interferences between this pulse and a reference beam. The probe wavelength is $618 \mathrm{~nm}$, the sample temperature is $300 \mathrm{~K}$. We use the fitting curve they have built from their experimental measurements,

We have plotted the phase shift predicted by the multigroup model and the temperature model [16] and the results measured in [14]. The results are shown in Fig. 14 for an intensity of $1.4 \mathrm{TW} / \mathrm{cm}^{2}$ and in Fig. 15 for an intensity of $2.1 \mathrm{TW} / \mathrm{cm}^{2}$.

From these two tests, we may conclude that the multigroup approach appears to be more accurate than the temperature model. However the central part of the phase shift differs a little bit between the multigroup approach and the results of [14].

With the multigroup model, collisional ionization only occurs once the higher energy level is populated. Thus for weak ionization, collisional ionization happens later than with the temperature model. In [14], the ex- 

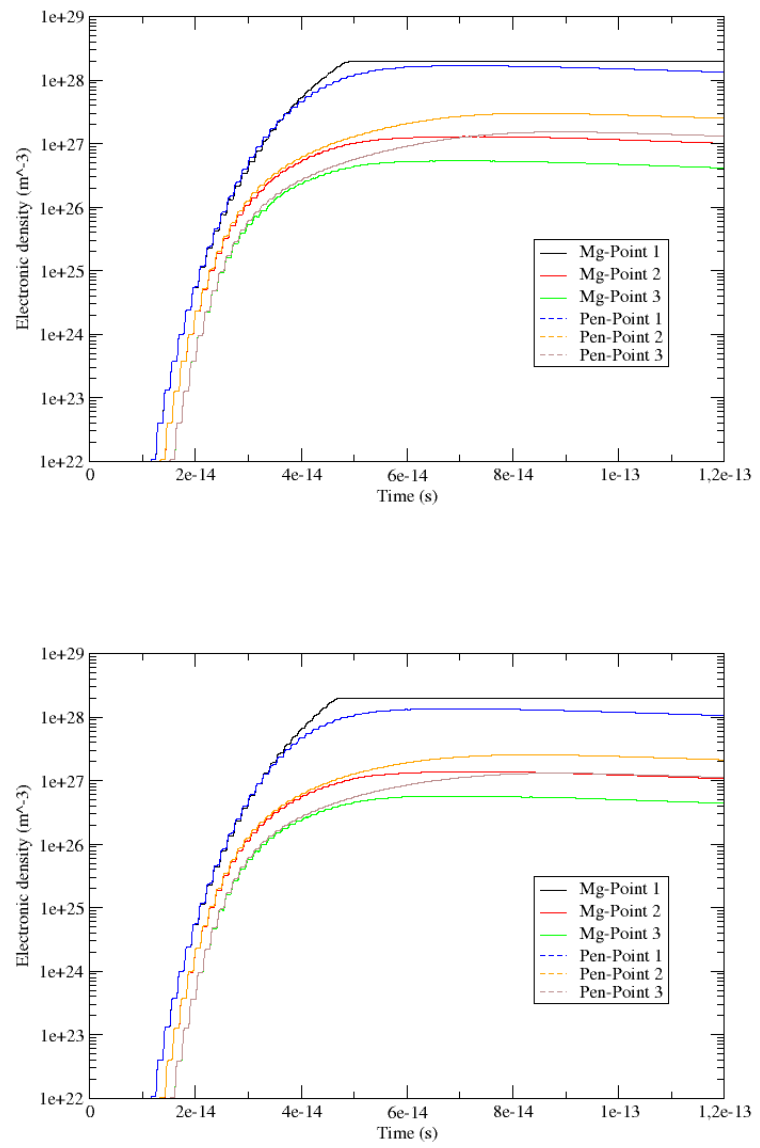

Figure 11: Total number of free electrons over time for the two models for three different points in the dielectric over time in 1D (first picture) and 3D (second picture). The first point is located at the front of the dielectric, the second one after $1.5 \mu \mathrm{m}$ and the last one at the back. 


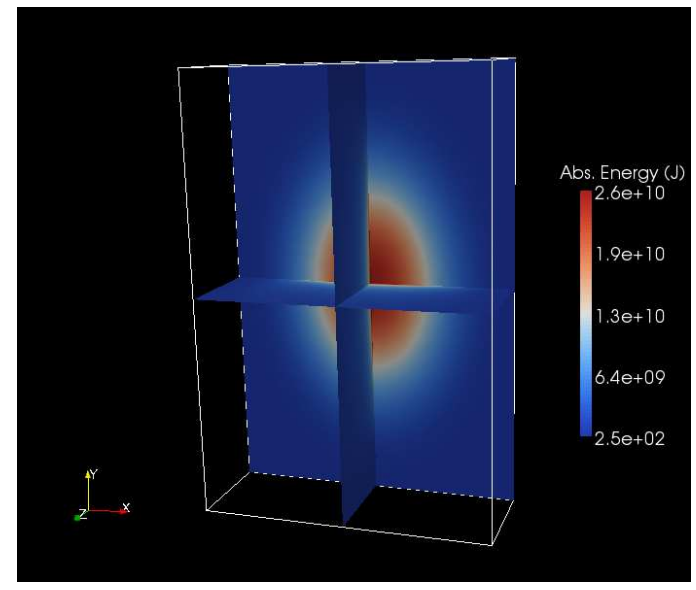

Figure 12: Absorbed energy for the temperature model. The absorbed energy is represented in J. The vertical slice at the back of the picture is located at $z=10^{-7} \mathrm{~m}$ of the front of the material. The two other slices are at the center of the $x$ and $y$ directions. The same color scale is used for the three slices.

perimental results are fitted with a model neglecting collisional ionization. For the lower intensity $1.4 \mathrm{TW} / \mathrm{cm}^{2}$ on Fig. 14, there is almost a perfect match between the results of [14] and our model.

\section{Conclusion}

We have presented a mathematical model for early ionization in dielectrics. It uses multiple-rate equations for unbound electrons and neutrals. The laser pulse is described by Maxwell equations. The cascading effect and ohmic heating are rendered by a procedure ensuring the conservation of energy.

Comparing to pre-existing works, we have a full time-space domain model (contrary to [18]) where we do not need an intuitive expression for the one-photon intraband absorption and using multiple rate equations we can render cascading effects (contrary to [16]).

The model was kept (relatively) simple : several phenomena, which we believe do not play necessarily an important role with ultrashort pulses, were neglected. For instance, the trapped electrons (accounted for by the term in $\alpha_{\text {des }}$ in Eq. (3)) are not set aside whereas they could be considered as excitons. We have also neglected the fact that when the highest energy 


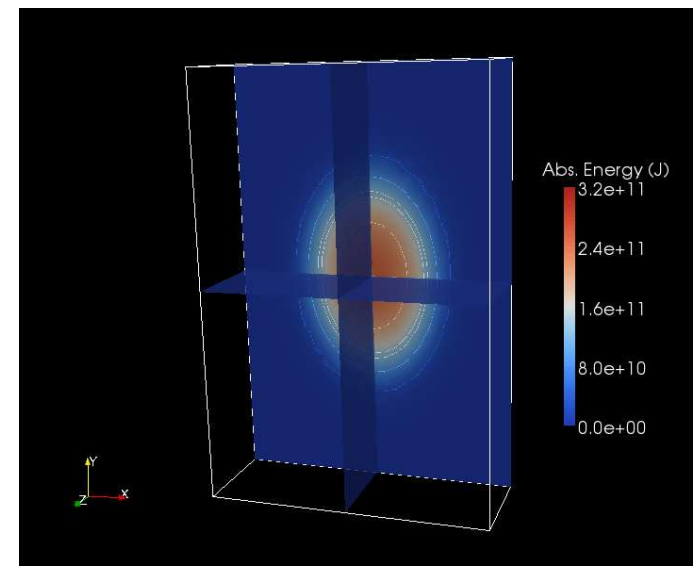

Figure 13: Absorbed energy for the multigroup model. The contours shown represents five uniformly distributed values ranging from $2.6 \times 10^{10} \mathrm{~J}$ (i.e. the maximum obtained with the temperature model) to $3.2 \times 10^{11} \mathrm{~J}$ (not included). The absorbed energy is represented in J. The vertical slice at the back of the picture is located at $z=10^{-7} \mathrm{~m}$ of the front of the material. The two other slices are at the center of the $x$ and $y$ directions. The same color scale is used for the three slices. 


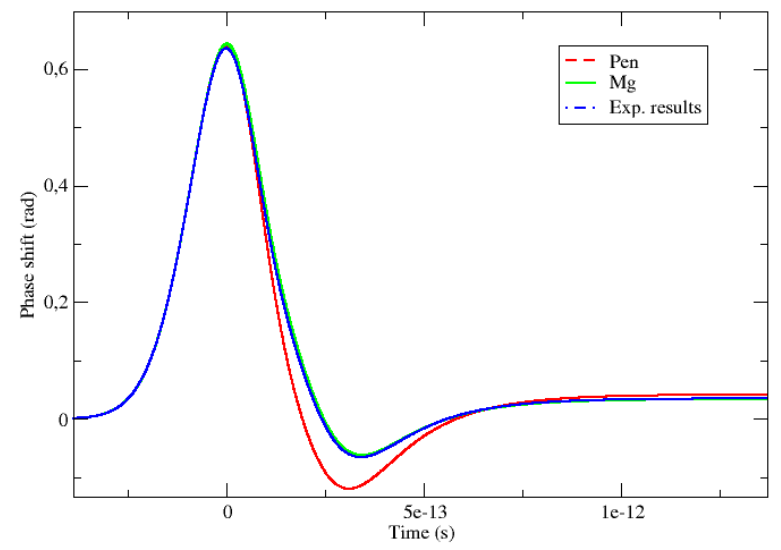

Figure 14: Phase shift (in rad) in $\mathrm{SiO}_{2}$ for the three approaches for an intensity of $1.4 \mathrm{TW} / \mathrm{cm}^{2}$. "Pen" denotes the curve obtained with the temperature model [16], "Mg" the curve obtained with the multigroup model described in this paper and "Exp. results" the results from [14]. 


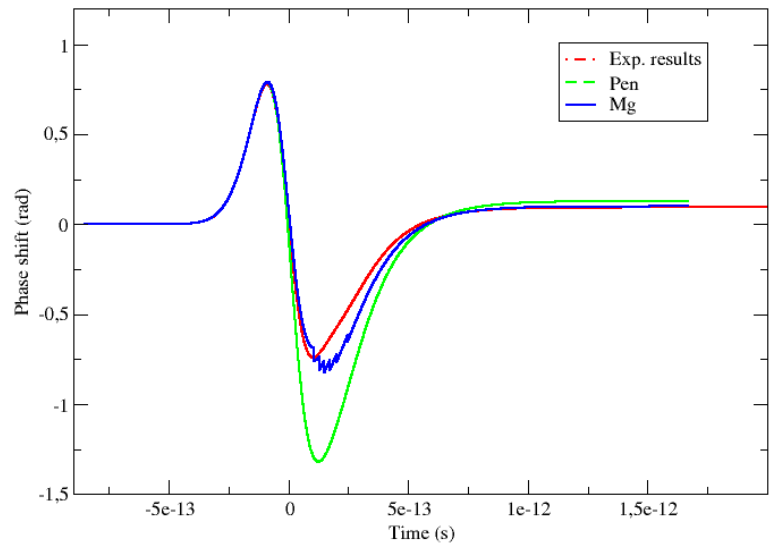

Figure 15: Phase shift (in rad) in $\mathrm{SiO}_{2}$ for the three approaches for an intensity of $2.1 \mathrm{TW} / \mathrm{cm}^{2}$. "Pen" denotes the curve obtained with the temperature model [16], "Mg" the curve obtained with the multigroup model described in this paper and "Exp. results" the results from [14]. 
level of the valence band is empty, the pulse can interact with the lower layers of the valence band to repopulate this level. However we believe that for the pulse durations we consider, the role of this phenomenon is probably quite small. Yet, this is the subject of a forthcoming work, which will also introduce tunneling ionization we have not considered here.

Some parameters that we have used are difficult to determine precisely. In particular the ionization rate $\sigma_{6}$ in Eq. (38) comes from [11] and the collisional rate $\alpha_{\text {ion }}$ in Eq. (37) is not rigorously justified. The results obtained also show that the observed phenomena depend on the model. The recovery of parameters has to be coupled to a specific model.

Even if our numerical results are qualitative, they show that with strong ionization, our multi-group approach yields different simulations that the temperature model [16]. This may prove that there is no electronic temperature. However, our model is much more computationally expensive than [16]. Our numerical codes are adapted to run on parallel architectures.

\section{References}

[1] T. Apostolova and Y. Hahn. Modeling of laser-induced breakdown in dielectrics with subpicosecond pulses. Journal of Applied Physics, $88: 1024,2000$.

[2] D. Arnold, E. Cartier, and D. J. DiMaria. Acoustic-phonon runaway and impact ionization by hot electrons in silicon dioxide. Phys. Rev. $B$, 45(3):1477-1480, Jan 1992.

[3] L. Bergé, S. Skupin, R. Nuter, J. Kasparian, and JP Wolf. Ultrashort filaments of light in weakly ionized, optically transparent media. Reports on Progress in Physics, 70:1633-1713, 2007.

[4] C. Besse, B. Bidégaray, A. Bourgeade, P. Degond, and O. Saut. A Maxwell-Bloch model with discrete symmetries for wave propagation in nonlinear crystals: an application to KDP. M2AN Math. Model. Numer. Anal., 38(2):321-344, 2004.

[5] A. Bourgeade and B. Nkonga. Dynamic Load Balancing Computation of Pulses Propagating in a Nonlinear Medium. The Journal of Supercomputing, 28(3):279-294, 2004.

[6] A. Bourgeade and O. Saut. Numerical methods for the bidimensional Maxwell-Bloch equations in nonlinear crystals. Journal of Computational Physics, 213(2):823-843, 2006.

[7] Robert W. Boyd. Nonlinear Optics. Academic Press, 1992. 
[8] BH Christensen and P. Balling. Modeling ultrashort-pulse laser ablation of dielectric materials. Physical Review B, 79(15):155424, 2009.

[9] A. Couairon and A. Mysyrowicz. Femtosecond filamentation in transparent media. Physics Reports, 441(2-4):47-189, 2007.

[10] A.P. Joglekar, H. Liu, GJ Spooner, E. Meyhöfer, G. Mourou, and AJ Hunt. A study of the deterministic character of optical damage by femtosecond laser pulses and applications to nanomachining. Applied Physics B: Lasers and Optics, 77(1):25-30, 2003.

[11] L.V. Keldysh. Ionization in the field of a strong electromagnetic wave. J. Exptl. Theoret. Phys., 20(5):8, Oct 1965.

[12] P.K. Kennedy, O.R. Div, and A.F.B. Brooks. A first-order model for computation of laser-induced breakdown thresholds in ocular and aqueous media. I. Theory. Quantum Electronics, IEEE Journal of, 31(12):2241-2249, 1995.

[13] S.S. Mao, F. Quéré, S. Guizard, X. Mao, RE Russo, G. Petite, and P. Martin. Dynamics of femtosecond laser interactions with dielectrics. Applied Physics A: Materials Science \& Processing, 79(7):1695-1709, 2004.

[14] P. Martin, S. Guizard, P. Daguzan, G. Petite, P. D'Oliveira, P. Meynadier, and M. Perdrix. Subpicosecond study of carrier trapping dynamics in wide-band-gap crystals. Physical Review B, 55(9):57995810, 1997.

[15] Alan C. Newell and Jerome V. Moloney. Nonlinear optics. Advanced Topics in the Interdisciplinary Mathematical Sciences. Addison-Wesley Publishing Company Advanced Book Program, Redwood City, CA, 1992.

[16] J.R. Peñano, P. Sprangle, B. Hafizi, W. Manheimer, and A. Zigler. Transmission of intense femtosecond laser pulses into dielectrics. Physical Review E, 72(3):36412, 2005.

[17] J. Qiu, K. Miura, and K. Hirao. Femtosecond laser-induced microfeatures in glasses and their applications. Journal of Non-Crystalline Solids, 2007.

[18] B. Rethfeld. Free-electron generation in laser-irradiated dielectrics. Physical Review B, 73(3):35101, 2006.

[19] B. Rethfeld, A. Kaiser, M. Vicanek, and G. Simon. Ultrafast dynamics of nonequilibrium electrons in metals under femtosecond laser irradiation. Physical Review B, 65(21):214303, 2002. 
[20] B. C. Stuart, M. D. Feit, S. Herman, A. M. Rubenchik, B. W. Shore, and M. D. Perry. Nanosecond-to-femtosecond laser-induced breakdown in dielectrics. Phys. Rev. B, 53(4):1749-1761, Jan 1996.

[21] SR Vatsya and SK Nikumb. Modeling of laser-induced avalanche in dielectrics. Journal of Applied Physics, 91:344, 2002.

[22] Y.V. White, X. Li, Z. Sikorski, L.M. Davis, and W. Hofmeister. Singlepulse ultrafast-laser machining of high aspect nano-holes at the surface of SiO_2. Optics Express, 16(19):14411-14420, 2008.

[23] K. S. Yee. Numerical solution of initial boundary value problems involving Maxwell's equations in isotropic media. IEEE Trans. Antennas Propag., AP-14:302-307, 1966. 